\title{
Role of V-V dimerization in the insulator-metal transition and optical transmittance of pure and doped $\mathrm{VO}_{2}$ thin films
}

\author{
S. S. Majid, ${ }^{1}$ S. R. Sahu, ${ }^{2}$ A. Ahad, ${ }^{1}$ K. Dey, ${ }^{2}$ K. Gautam, ${ }^{2}$ F. Rahman, ${ }^{1}$ \\ P. Behera, ${ }^{2}$ U. Deshpande, ${ }^{2}$ V. G. Sathe, ${ }^{2}$ and D. K. Shukla ${ }^{2, *}$ \\ ${ }^{1}$ Department of Physics, Aligarh Muslim University, Aligarh-202002, India \\ ${ }^{2}$ UGC-DAE Consortium for Scientific Research, Indore-452001, India
}

(Received 14 June 2019; revised manuscript received 5 December 2019; published 17 January 2020)

\begin{abstract}
An insulator to metal (IMT) transition $\left(T_{t} \sim 341 \mathrm{~K}\right)$ in the $\mathrm{VO}_{2}$ accompanies a transition from an infrared (IR) transparent to IR opaque phase. Tailoring of the IMT and associated IR switching behavior can offer potential thermochromic applications. Here we report on effects of the $\mathrm{W}$ and the Tb doping on the IMT and associated structural, electronic structure, and optical properties of the $\mathrm{VO}_{2}$ thin film. Our results show that the W doping significantly lowers IMT temperature $(\sim 292 \mathrm{~K}$ to $\sim 247 \mathrm{~K}$ for $1.3 \% \mathrm{~W}$ to $3.7 \% \mathrm{~W})$ by stabilizing the metallic rutile, $R$, phase while Tb doping does not alter the IMT temperature much and retains the insulating monoclinic, $M 1$, phase at room temperature. It is observed that the $\mathrm{W}$ doping albeit significantly reduces the IR switching temperature but is detrimental to the solar modulation ability, contrary to the Tb doping effects where higher IR switching temperature and solar modulation ability is observed. The IMT behavior, electrical conductivity, and IR switching behavior in the $\mathrm{W}$ and the Tb-doped thin films are found to be directly associated with the spectral changes in the $\mathrm{V} 3 d_{\|}$states.
\end{abstract}

DOI: 10.1103/PhysRevB.101.014108

\section{INTRODUCTION}

Vanadium dioxide $\left(\mathrm{VO}_{2}\right)$ is known to undergo first-order insulator to metal transition (IMT) from the low-temperature monoclinic insulating phase $\left(P 2_{1} / c\right)$ to the high temperature rutile metallic $\left(\mathrm{PH}_{2} / \mathrm{mnm}\right)$ phase around the transition temperature $T_{t} \sim 341 \mathrm{~K}$. Structural transformation of $\mathrm{VO}_{2}$ from the rutile, $R$, structure to the monoclinic, $M 1$ structure transforms the equally spaced $\mathrm{V}$ atoms, oriented along the rutile $c$ axis, into paired (dimerized) and tilted $\mathrm{V}$ atoms in the monoclinic structure [1-3]. The IMT in $\mathrm{VO}_{2}$ is accompanied by a dramatic modification in optical properties like switching in transmittance/reflectance of the IR radiations with the insulating phase being transparent to the IR while the metallic phase blocks them. This property of $\mathrm{VO}_{2}$ is potentially useful for designing thermochromic smart windows which can serve as the best energy-management devices as these windows switch the solar heat gain [4-6].

Changes in the IR and visible transmittance of the $\mathrm{VO}_{2}$ thin films across the IMT can be explained in terms of changes in the band structures, explained in context of the Goodenough model $[7,8]$. According to this model, in the rutile metallic phase of the $\mathrm{VO}_{2}$ the octahedral crystal field splits the $\mathrm{V}$ $3 d$ degenerate orbitals into doubly degenerate $e_{g}^{\sigma}$ and triply degenerate $t_{2 g}$ orbitals. The $t_{2 g}$ orbitals are further split into the doubly degenerate $e_{g}^{\pi}$ orbitals and the single $d_{x^{2}-y^{2}}\left(\mathrm{~d}_{\|}\right)$ orbital. Both the $e_{g}^{\pi}$ and $e_{g}^{\sigma}$ states hybridize with $\mathrm{O} 2 p$ orbitals to form antibonding $\pi^{*}$ and $\sigma^{*}$ molecular orbitals while the $\mathrm{d}_{\|}$orbitals are oriented along the rutile $c$ axis. In the

*dkshukla@csr.res.in insulating phase, pairing and tilting of the vanadium atoms along the rutile $c$ axis results in splitting of the $\mathrm{d}_{\|}$orbitals into filled bonding and empty antibonding orbitals. A band gap of $\sim 0.65 \mathrm{eV}$ in the $\mathrm{VO}_{2}$ insulating phase arises due to energy differences between the bonding $\mathrm{V} 3 d_{\|}$and the $\pi^{*}$ bands while, in the metallic phase, the energy gap vanishes due to overlapping of the nonbonding $3 d_{\|}$and $\pi^{*}$ bands at the Fermi level. Decrease in the IR transmittance of the $\mathrm{VO}_{2}$ with increase in temperature is directly associated with vanishing of the $\mathrm{VO}_{2}$ insulating band gap $(\sim 0.65 \mathrm{eV})$ and increase in the free carrier concentration in the high-temperature metallic phase. Optical transitions in the visible spectral range occur between lower $\mathrm{O} 2 p$ valence states to the $\pi^{*}$ conduction bands $[9,10]$.

For practical applications of the $\mathrm{VO}_{2}$ material such as thermochromic smart windows, the IMT temperature of the $\mathrm{VO}_{2}$ requires that it be tailored down to room temperature (RT). Chemical doping of electron-rich valence shell metal ions viz. $\mathrm{W}^{6+}, \mathrm{Mo}^{6+}$, and $\mathrm{Nb}^{6+}$ and optimizing the amount of strain in epitaxial $\mathrm{VO}_{2}$ thin films have been found to be effective ways to reduce the $T_{t}$ of the $\mathrm{VO}_{2}$ to RT [9,1114]. Doping of electron-doped ions tungsten $\left(\mathrm{W}^{6+}\right)$ have been found effective in reducing the transition temperature to RT, while doping of the hole-doped ions terbium $\left(\mathrm{Tb}^{3+}\right)$ has no significant impact on the transition temperature lowering [15]. There is always a trade-off between the transition temperature reduction, solar modulation ability, and the luminous transmittance in $\mathrm{VO}_{2}$ thin films [6]. $\mathrm{W}$ doping is found to reduce the solar modulation ability and the luminous transmittance $[16,17]$ while $\mathrm{Tb}$ doping enhances the luminous transmittance accompanied by the decrease in the solar modulation ability [15]. Two systems (W- and Tb-doped $\mathrm{VO}_{2}$ ) show contrasting 
effects on the electrical and optical properties of the $\mathrm{VO}_{2}$ thin films. It is essential to completely study both the $\mathrm{W}$ and the $\mathrm{Tb}$ $\mathrm{VO}_{2}$ systems and explore the role of $\mathrm{W}$ and $\mathrm{Tb}$ in modifying the $\mathrm{VO}_{2} \mathrm{IMT}$ and optical properties.

We have synthesized the pure $\mathrm{VO}_{2}$ and $\mathrm{W}$ - $(1.3 \%, 2.9 \%$, and $3.7 \%)$ and $\mathrm{Tb}-(1.3 \%, 3.3 \%$, and $4.6 \%)$ doped $\mathrm{VO}_{2}$ thin films on the quartz substrates using the pulsed laser deposition technique. By combining structural, electrical, optical, and electronic structure studies, we present an insightful of the effects of the $\mathrm{W}$ and $\mathrm{Tb}$ doping on the IMT and optical properties of the $\mathrm{VO}_{2}$. Our results directly show the dopinginduced alterations to the $\mathrm{V}-\mathrm{V}$ dimerization in the $\mathrm{VO}_{2}$ thin film, on which electrical and optical properties are largely dependent.

\section{EXPERIMENTAL DETAILS}

$\operatorname{KrF}$ excimer laser $(\lambda=248 \mathrm{~nm}$, repetition rate of $5 \mathrm{~Hz}$ and pulse energy of $210 \mathrm{~mJ}$ ) was focused onto a target (pressed pure, $\mathrm{W}$ - and Tb-doped $\mathrm{V}_{2} \mathrm{O}_{5}$ ) with a fluence of $1.1 \mathrm{~J} / \mathrm{cm}^{2}$. Ultrasonically cleaned quartz substrates were maintained at a temperature of $700{ }^{\circ} \mathrm{C}$ during the deposition. All depositions were performed in an oxygen partial pressure of 10 mTorr. Xray diffraction (XRD) was carried out with a Bruker D8 x-ray diffractometer with $\mathrm{Cu} \mathrm{K} \alpha$ radiation. Temperature-dependent resistivity measurements were performed in the standard fourpoint configuration. Temperature-dependent Raman spectra were collected in backscattering geometry using a $10 \mathrm{~mW}$ $\operatorname{Ar}(475 \mathrm{~nm})$ laser as an excitation source coupled with a Labram-HRF micro-Raman spectrometer equipped with a 50X objective. The x-ray photoelectron spectroscopy (XPS) measurements were performed using an Omicron energy analyzer (EA-125) with a $\mathrm{Al} \mathrm{K} \mathrm{K}_{\alpha}(1486.6 \mathrm{eV})$ x-ray source. Soft x-ray absorption spectroscopy (SXAS) across the $\mathrm{V} L_{3,2}$ and the $\mathrm{O} K$ edges were carried out in the total electron yield mode at the beamline BL-01, Indus-2 at RRCAT, Indore, India. Energy resolution during SXAS measurements at the oxygen $K$-edge energy was $\sim 250 \mathrm{meV}$. Bulk $\mathrm{VO}_{2}$ was measured for reference. $\mathrm{V}$ - $K$-edge $\mathrm{x}$-ray absorption near edge spectroscopy measurements were performed at beamline P64, PETRA III, DESY, Hamburg, Germany in fluorescence mode. Optical transmittance spectra were collected using UV-VISNIR spectrometer, Perkin Elmer USA Model: Lambda 950, operating in the spectral range of 175 to $3300 \mathrm{~nm}$ having resolution $\sim 0.02 \mathrm{~nm}$ (UV-VIS) and $\sim 0.2 \mathrm{~nm}$ (NIR). Doping percentages used throughout the paper are calculated using the XPS spectra of $\mathrm{Tb}$ and $\mathrm{W}$.

\section{RESULTS AND DISCUSSIONS}

Figure 1(a) shows XRD of pure and W-doped thin films. XRD confirms growth of the $\mathrm{VO}_{2}$ monoclinic (MI) phase in the undoped thin films, visualized from the diffraction peaks observed at $2 \theta \sim 27.97^{\circ}$ and $57.79^{\circ}$, corresponding to the reflections from the $\mathrm{VO}_{2}$ monoclinic $\left(\begin{array}{lll}0 & 1 & 1\end{array}\right)$ and $(0$ 2 2) planes, respectively $[18,19]$. Tungsten-doped $\mathrm{VO}_{2}$ thin films are grown in the rutile $(R)$ phase, evident from $2 \theta$ positions of the diffraction peaks at $\sim 27.78^{\circ}$ and $57.27^{\circ}$, which correspond to reflections from the rutile $\left(\begin{array}{lll}1 & 1 & 0\end{array}\right)$ and
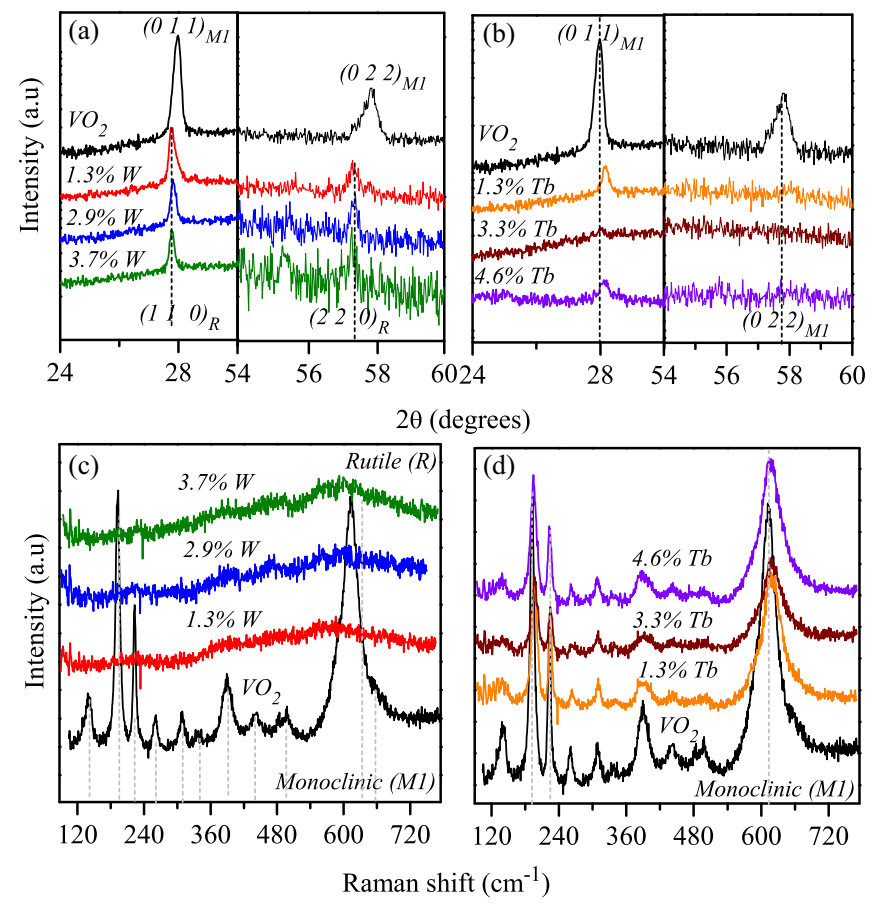

FIG. 1. Room-temperature XRD patterns of (a) pure and Wdoped $\mathrm{VO}_{2}$ thin films, (b) pure and Tb-doped $\mathrm{VO}_{2}$ thin films. Room temperature Raman spectra of (c) pure and $\mathrm{W}$-doped $\mathrm{VO}_{2}$ thin films and (d) pure and Tb-doped $\mathrm{VO}_{2}$ thin films.

(2 2 0) planes, respectively [19]. Further confirmation about grown phases in pure and $\mathrm{W}$-doped $\mathrm{VO}_{2}$ thin films comes from the Raman spectra shown in the Fig. 1(c). The pure $\mathrm{VO}_{2}$ thin film shows seven $\mathrm{A}_{g}$ and three $\mathrm{B}_{g}$ Raman modes at $\sim 139$, $192,222,309,337,389,613 \mathrm{~cm}^{-1}$ and $260,441,496 \mathrm{~cm}^{-1}$, respectively, which belong to the $\mathrm{VO}_{2}$ monoclinic, $\mathrm{M} 1$, phase [20]. The $\mathrm{A}_{g}$ symmetry Raman modes $\omega_{v_{1}}$ and $\omega_{v_{2}}$ at $\sim 192$ and $222 \mathrm{~cm}^{-1}$ are assigned to the V-V vibrations while the rest of the observed Raman peaks are related to V-O vibrations [21,22]. Complete disappearance of the $M 1$ vibrational modes observed in all W-doped thin films confirm growth of the rutile phase, consistent with earlier reports [18,23]. XRD patterns of the Tb-doped thin films shown in Fig. 1(b) exhibit a slight decrease in crystalline nature and shifting of the monoclinic, $M 1,\left(\begin{array}{lll}0 & 1 & 1\end{array}\right)$ diffraction peak toward higher $2 \theta$, compared to the pure $\mathrm{VO}_{2}$ thin film. Shifting of the diffraction peaks to higher $2 \theta$ values with increase in the Tb-doping percentage is attributed to decrease in unit cell size of the $\mathrm{VO}_{2}$. The nonproportionality of $\left(\begin{array}{lll}0 & 1 & 1\end{array}\right)$ intensity with $\mathrm{Tb}$ doping may be attributed to the misalignment of the 3.3\% Tb-doped sample during the XRD measurements, which is clear from the background behavior too. XRD studies reveal the highly oriented growth of the $\mathrm{VO}_{2}$ thin films on the amorphous quartz substrate. Raman spectra shown in Fig. 1(d) further confirms growth of the monoclinic, $M 1$, phase in the Tb-doped thin films and substantiates to XRD results. In contrast to the $\mathrm{W}$ doping, no phase transformation is visualized due to the $\mathrm{Tb}$ doping.

Temperature-dependent resistivity $(\rho)$ measurements of the pure and the $\mathrm{W}-/ \mathrm{Tb}$-doped $\mathrm{VO}_{2}$ thin films are shown 


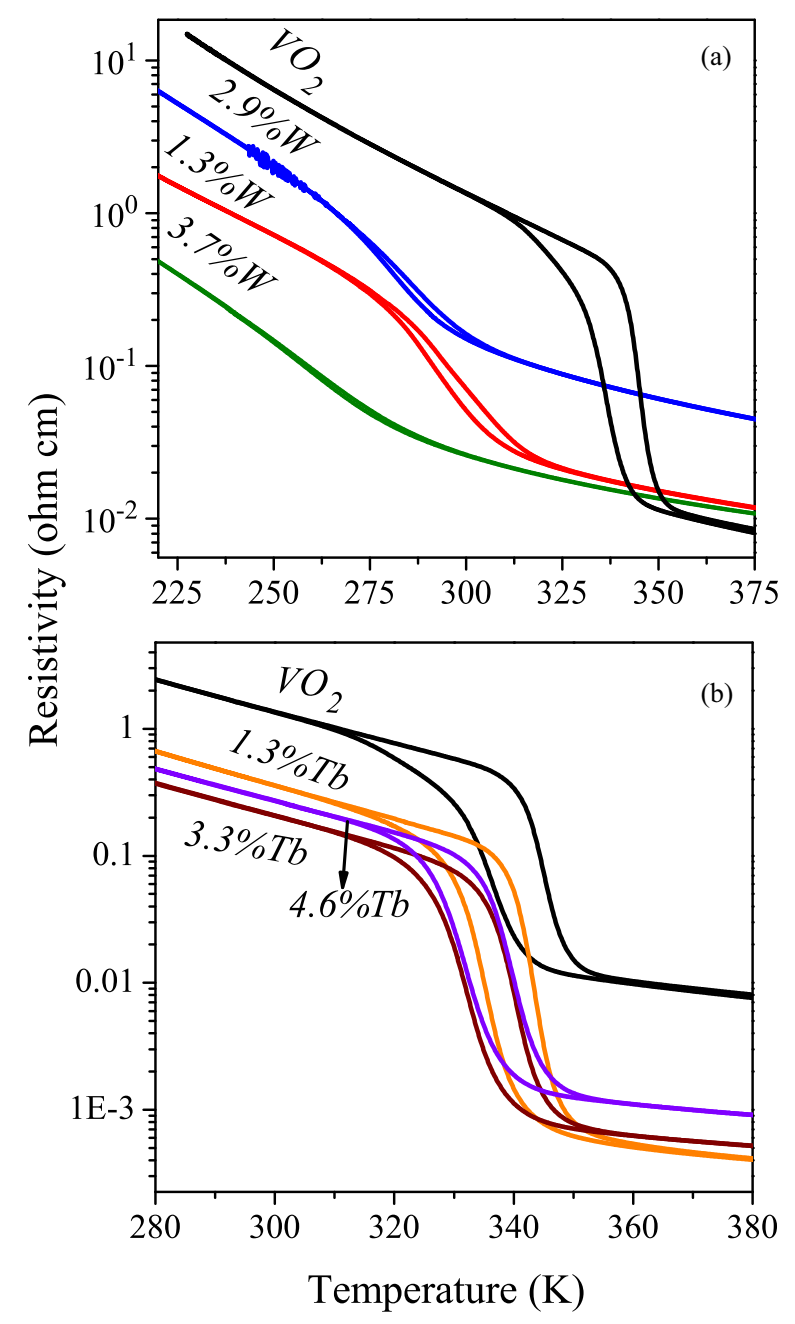

FIG. 2. Temperature-dependent resistivity of (a) pure and Wdoped $\mathrm{VO}_{2}$ thin films (b) pure and Tb-doped $\mathrm{VO}_{2}$ thin films.

in Figs. 2(a) and 2(b). The characteristic IMT is observed in all the thin films. To infer quantitative information from temperature-dependent resistivity measurements, the IMT in all the thin films were fitted by the Boltzmann function $\left[f(T)=1-\frac{1}{1+e^{\frac{T-T_{0}}{d T}}}\right.$ Eq. (1)]. $f$ is the resistivity, $(\rho), T_{0}$ is the transition temperature and dT signifies the transition width. IMT temperature $\left(T_{t}\right)$ is defined as an average of transition temperatures measured in the cooling and the heating cycles. The IMT temperature for the pure and doped $\mathrm{VO}_{2}$ thin films are illustrated in Table I. Tungsten doping has effectively reduced the IMT transition temperature of the $\mathrm{VO}_{2}$ thin film. In the $\sim 1.3 \% \mathrm{~W}$-doped $\mathrm{VO}_{2}$ thin film, IMT is observed near RT $(\sim 292 \mathrm{~K})$, which is consistent with the earlier reports $[24,25]$. Tb doping has small impact on the IMT temperature of the $\mathrm{VO}_{2}$ thin film compared to the $\mathrm{W}$ doping. Resistivity measurements are also used to calculate the IMT strength defined as resistivity ratio $\rho\left(T_{t}\right.$-dT $) / \rho\left(T_{t}+\mathrm{dT}\right)$. Calculated values of the IMT strength of the pure and the doped $\mathrm{VO}_{2}$ thin films are listed in Table I.

For the correct estimation of chemical states of the vanadium and the dopant elements, we carried out XPS measurements, shown in Fig. 3. XPS of the pure $\mathrm{VO}_{2}$ thin film [Fig. 3(a)] confirms the +4 oxidation state of the vanadium, which is visible from the binding energy positions of the spinorbit split features V $2 p_{3 / 2} \sim 516 \mathrm{eV}$ and $\mathrm{V} 2 p_{1 / 2} \sim 523 \mathrm{eV}$. The features at $\sim 531.3 \mathrm{eV}, \sim 530 \mathrm{eV}$, and $\sim 527 \mathrm{eV}$ correspond to the $\mathrm{OH}$ concentration, $\mathrm{O} 1 s$, and the $\mathrm{V} 2 p_{3 / 2}$ satellite peak, respectively $[26,27]$. $\mathrm{VO}_{2}$ contains satellite features for both the $\mathrm{V} 2 p_{3 / 2}$ and $\mathrm{V} 2 p_{1 / 2}$ states due to strong hybridization between the $\mathrm{V} 3 d$ and the $\mathrm{O} 2 p$ states [27]. The $\mathrm{V} 2 p_{3 / 2}$ satellite peak, $\mathrm{S} 1, \sim 527 \mathrm{eV}$ is found in between the $\mathrm{V} 2 p_{1 / 2}$ and $\mathrm{O} 1 s$ spectral features while $\mathrm{V} 2 p_{1 / 2}$ satellite peak is located on the higher binding energy side of $\mathrm{O} 1 s$ peak and is mixed with the states corresponding to the $\mathrm{OH}$ concentration. The spectral feature, $\mathrm{S} 2$, at $\sim 520 \mathrm{eV}$ is assigned to X-ray satellite peak of $\mathrm{Al} \mathrm{K}_{\alpha 3}$ source and arises from a nonmonochromatic $x$-ray radiation source [28]. W doping [Figs. 3(b) and 3(c)] induces the spectral features at binding energies $\sim 514 \mathrm{eV}$ and $\sim 522 \mathrm{eV}$, belonging to $V^{3+} 2 p_{3 / 2}$ and $2 p_{1 / 2}$ states, while Tb [Figs. 3(d)-3(f)] doping results in the inclusion of $V^{5+} 2 p_{3 / 2}$ and $V^{5+} 2 p_{1 / 2}$ states at the binding energy values $\sim 517 \mathrm{eV}$ and $\sim 524 \mathrm{eV}$. XPS spectra shown in Figs. 4(a)-4(c) confirm the +6 oxidation state of the $\mathrm{W}$ which is evident from the binding energy positions of $4 f_{7 / 2} \sim 35 \mathrm{eV}$ and $4 f_{5 / 2} \sim 37 \mathrm{eV}$ features, while Figs. 4(d) -4 (f) represent +3 oxidation state of the $\mathrm{Tb}$ which is visualized from the spectral features at $\sim 1241 \mathrm{eV}$ and $\sim 1276 \mathrm{eV}$ belonging to $\mathrm{Tb} 3 d_{5 / 2}$ and $3 f_{3 / 2}$ states. The multiple valency of vanadium states is difficult to observe from the V $3 p$ XPS spectra because of the strong multiplet splitting due to the large spatial overlap with the $\mathrm{V} 3 d$ shell [26], hence the feature at $\sim 41.5 \mathrm{eV}$ is assigned to V $3 p$ states, consistent with earlier reports [15,29].

TABLE I. Parameters $T_{t}, T_{s c}, T_{o c}$ and IMT strength obtained from the temperature-dependent resistivity, Raman (cooling cycle), and transmittance spectroscopy (cooling cycle) in pure and doped $\mathrm{VO}_{2}$ thin films. The $d_{\|}$intensity in the pure and doped $\mathrm{VO}_{2}$ thin films is manifested from the XAS measurements.

\begin{tabular}{|c|c|c|c|c|c|}
\hline Sample & $\begin{array}{l}\quad T_{t}(K) \\
\text { resistivity }\end{array}$ & $\begin{array}{l}T_{s c}(K) \\
\text { Raman }\end{array}$ & $\begin{array}{c}T_{o c}(K) \\
\text { transmittance }\end{array}$ & $\begin{array}{l}\text { IMT strength } \\
\text { resistivity }\end{array}$ & $\begin{array}{c}d_{\|} \text {intensity } \\
\text { XAS }\end{array}$ \\
\hline $\mathrm{VO}_{2}$ & 340.1 & 322.3 & 320.7 & 56.6 & $0.55 \pm 0.03$ \\
\hline $2.9 \% \mathrm{~W}$ & 275.8 & 198.9 & 246.2 & 18.8 & $0.45 \pm 0.03$ \\
\hline $3.7 \% \mathrm{~W}$ & 247.3 & 122.5 & 217.1 & 11.1 & $0.46 \pm 0.03$ \\
\hline $1.3 \% \mathrm{~Tb}$ & 339.2 & 329.3 & 341.6 & 271.4 & $0.64 \pm 0.06$ \\
\hline $4.6 \% \mathrm{~Tb}$ & 335.5 & 322.3 & 330.5 & 109.9 & $0.66 \pm 0.06$ \\
\hline
\end{tabular}




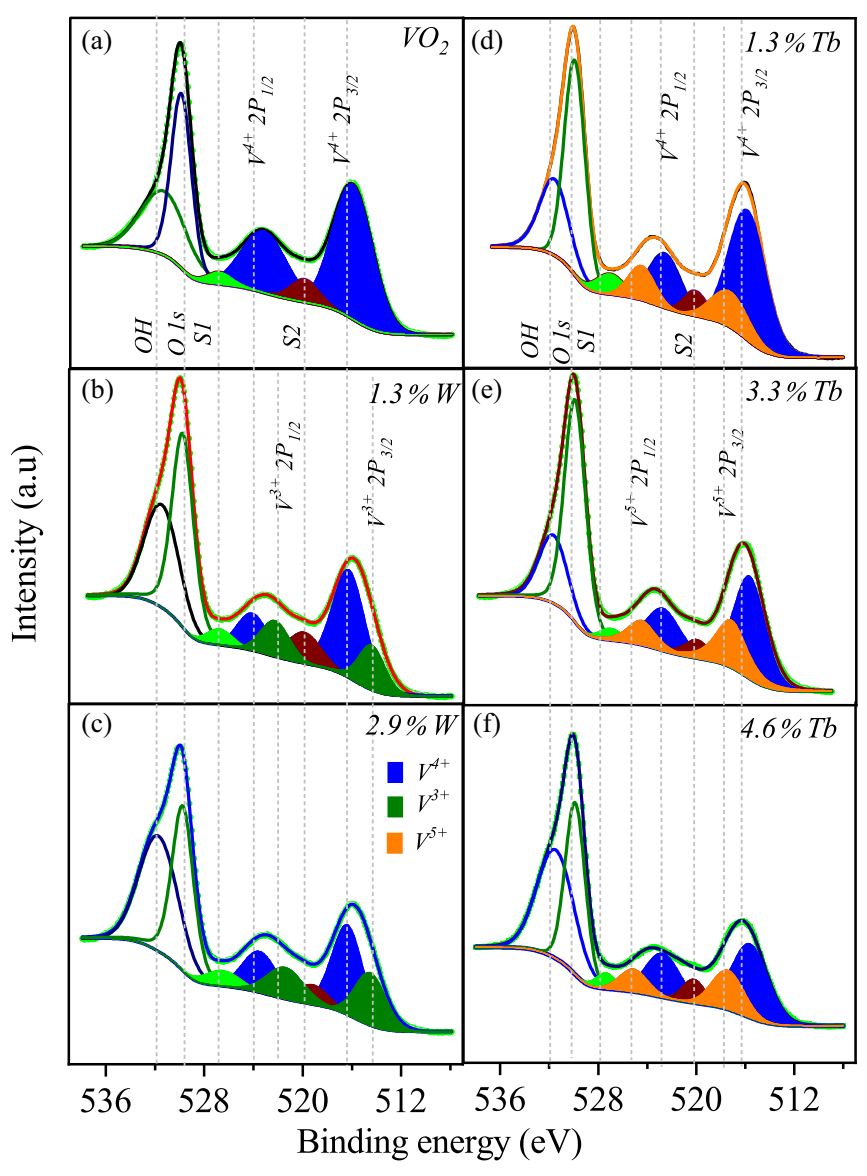

FIG. 3. O $1 s$ and V $2 p$ XPS of (a) the pure $\mathrm{VO}_{2}$ (b) $1.3 \% \mathrm{~W}$, (c) $2.9 \% \mathrm{~W}$, (d) $1.3 \% \mathrm{~Tb}$, (e) $3.3 \% \mathrm{~Tb}$, and (f) $4.6 \% \mathrm{~Tb}$-doped $\mathrm{VO}_{2}$ thin films

To maintain charge neutrality in the $\mathrm{W}$ and Tb-doped $\mathrm{VO}_{2}$ thin films, some vanadium atoms change their oxidation state to +3 and +5 to compensate the higher $\left(W^{6+}\right)$ and the lower $\left(\mathrm{Tb}^{3+}\right)$ charge states. Figure $4(\mathrm{~g})$ shows a decrease in the $\mathrm{V}^{4+}$ percentage content with an increase in the doping percentage of the $\mathrm{W}$ and $\mathrm{Tb}$ atoms and the decrease in the $\mathrm{V}^{4+}$ content is found to be accompanied by an increase in the contents of the $V^{3+}$ and $V^{5+}$ for the $\mathrm{W}$ - and $\mathrm{Tb}$-doped $\mathrm{VO}_{2}$, respectively.

Temperature-dependent Raman spectra of the pure and the $\mathrm{W}$-/Tb-doped $\mathrm{VO}_{2}$ thin films are shown in Figs. 5(a)$5(\mathrm{~g})$, measured in the cooling cycles. Temperature-dependent structural transition from the low-temperature monoclinic $(M I)$ structure to the high-temperature rutile $(R)$ structure is observed in all the thin films without presence of any intermediate structures ( $M 2$ and $T$ ) which are usually accessible via chemical doping in the $\mathrm{VO}_{2}$ [18]. Temperature-dependent frequency position of the Raman mode $\omega_{0}$, of the pure and the W-/Tb-doped $\mathrm{VO}_{2}$ thin films are plotted in Figs. 5(h) and 5(i). There is not any anomalous change observed in the temperature-dependent frequency position of the Raman mode, $\omega_{0}$. Softening of the Raman mode positions due to lattice thermal expansion is accompanied by a large and sudden change at the structural transition temperature. In the Tb-doped $\mathrm{VO}_{2}$ thin films, frequency shift of the $\omega_{0}$ mode from $\sim 613 \mathrm{~cm}^{-1}$ (observed in the pure $\mathrm{VO}_{2}$ thin film, to
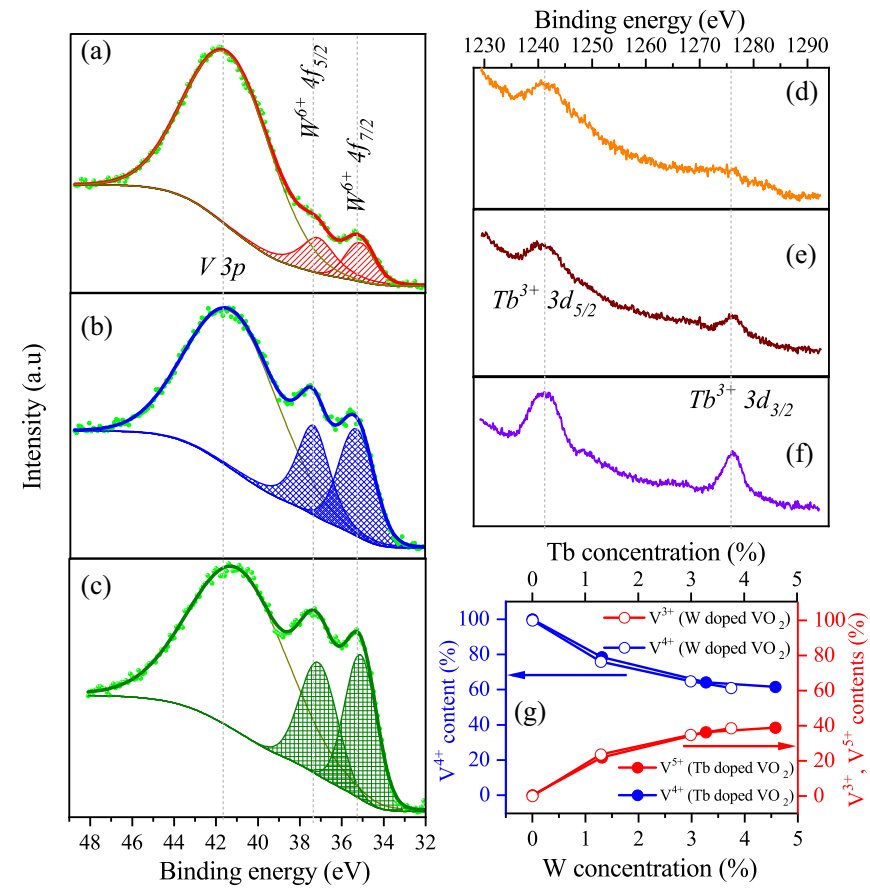

FIG. 4. (a)-(c) W $4 f$ XPS of the $1.3 \%, 2.9 \%$, and $3.7 \%$ W-doped $\mathrm{VO}_{2}$ thin films. (d)-(f) $\mathrm{Tb} 3 d$ XPS of the $1.3 \%, 3.3 \%$, and $4.6 \%$ Tb-doped $\mathrm{VO}_{2}$ thin films. (g) Variation of the $V^{5+}$ and $V^{3+}$ contents for the $\mathrm{Tb}$ - and $\mathrm{W}$-doping concentrations, along with the doping dependent $V^{4+}$ content variation in the $\mathrm{VO}_{2}$ thin film.

$\sim 619 \mathrm{~cm}^{-1}$ ) reflects significant influence of the Tb doping on the V-O vibrational mode compared to the $\mathrm{W}$ doping, where the $\omega_{0}$ mode stays around $\sim 613 \mathrm{~cm}^{-1}$. Normally, the higher frequency shift of the $\omega_{0}$ mode position in the pure $\mathrm{VO}_{2}$ thin film is considered a fingerprint for identification of the $\mathrm{VO}_{2} M 2$ and $T$ structures [18,21,30]; however, in the Tbdoped $\mathrm{VO}_{2}$ thin films, higher frequency shift of the $\omega_{0}$ mode to $\sim 619 \mathrm{~cm}^{-1}$ is much less compared to that observed for the $T$ phase $\left(\sim 629 \mathrm{~cm}^{-1}\right)$ and for the $M 2$ phase $\left(\sim 642 \mathrm{~cm}^{-1}\right)$.

Recently, Kalcheim et al. [31] used XRD and IR spectroscopic techniques to understand coupling between the IMT and the structural phase transition in the $\mathrm{V}_{2} \mathrm{O}_{3}$ thin films and discussed the nonreliability of the resistance measurements in determination of the correct insulating and metallic phase fractions. We have extracted the the monoclinic phase fraction (MPF) and the insulating phase fraction (IPF) from temperature-dependent Raman and optical transmittance spectra (discussed later, see Fig. 7), respectively. The following formula has been used to calculate the IPF (in the range, $2000 \mathrm{~cm}^{-1}$ to $2500 \mathrm{~cm}^{-1}$ ).

$$
\operatorname{IPF}(T)=\frac{T_{r}(T)-T_{r M}}{T_{r I}-T_{r M}}
$$

where $T_{r I}$ and $T_{r M}$ are transmittance of the pure insulating and the pure metallic phases and $T_{r}(T)$ is the transmittance at temperature, $T$. The calculated IPF of the pure and the $\mathrm{W}$-/Tb-doped $\mathrm{VO}_{2}$ thin films are compared with the MPF obtained from temperature-dependent integrated intensity of the $\omega_{0}$ Raman mode in Figs. 6(a)-6(f). Calculated transition temperatures, $T_{o c}$ and $T_{s c}$, from the optical transmittance 
Temperature $(\mathrm{K})$
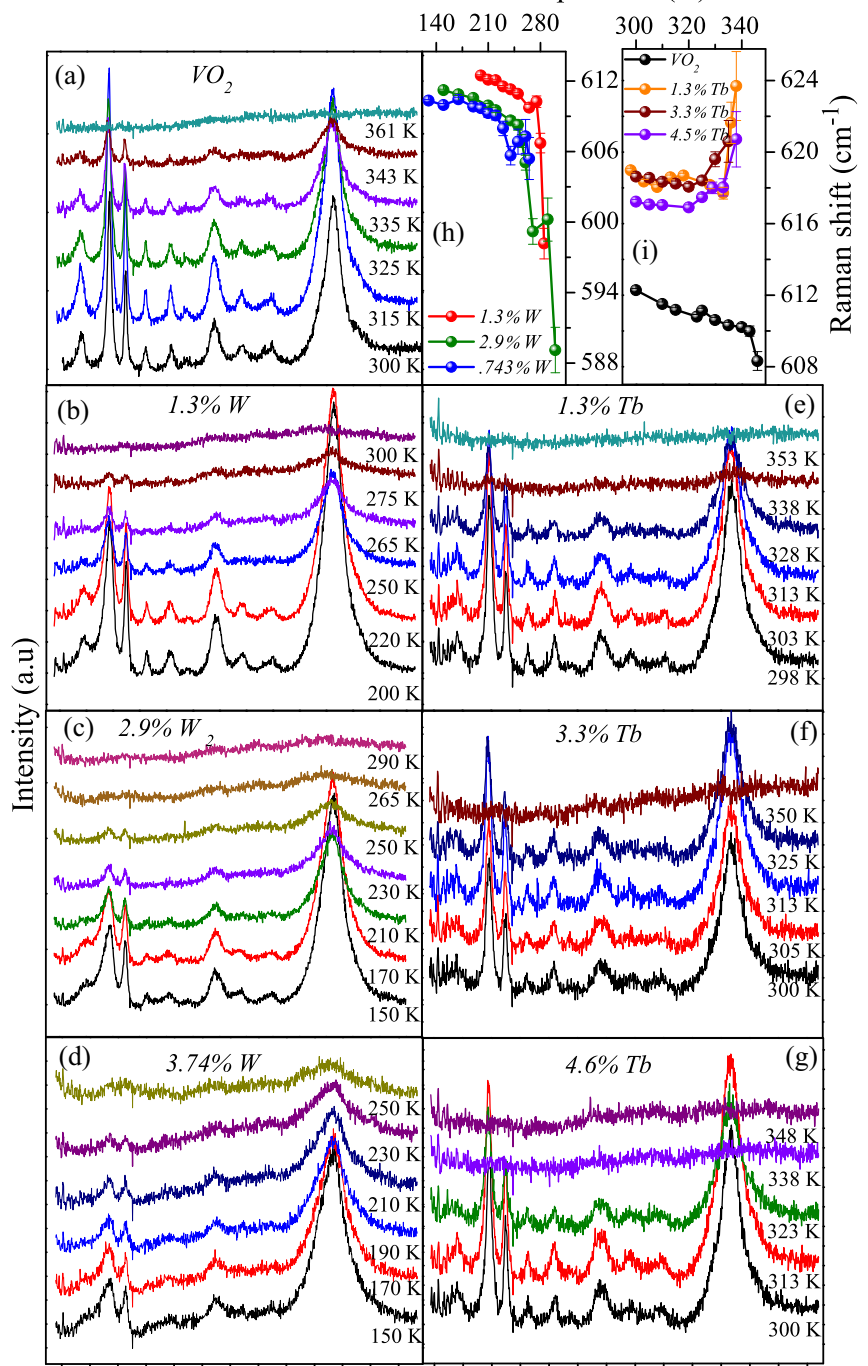

$\begin{array}{lllllllllllll}105 & 210 & 315 & 420 & 525 & 630 & 735100 & 200 & 300 & 400 & 500 & 600 & 700\end{array}$ Raman shift $\left(\mathrm{cm}^{-1}\right)$

FIG. 5. (a)-(g) Temperature-dependent Raman spectra of the pure and the $\mathrm{W}$-/Tb-doped $\mathrm{VO}_{2}$ thin films collected in the cooling cycle. Temperature-dependent frequency shift of the $\omega_{0}$ Raman mode positions obtained from Lorentz fitting in (h) W-doped (i) pure, and Tb-doped $\mathrm{VO}_{2}$ thin films.

measurement and the Raman spectroscopy data, using Eq. (1), are listed in Table I. In the pure $\mathrm{VO}_{2}$ thin film, IMT is found to be coupled with the structural transition while $\mathrm{W}$ and $\mathrm{Tb}$ doping are found to stabilize the rutile structure prior to the metallic state. Impact of the $\mathrm{W}$ doping in the rutile structure stabilization is much more significant compared to the $\mathrm{Tb}$ doping.

For thermochromic applications, optical properties of the pure and the doped $\mathrm{VO}_{2}$ thin films are characterized using temperature-dependent UV-VIS-NIR transmittance spectra shown in Fig. 7. The spectral transmittance in the wavelength range of $\sim 250 \mathrm{~nm}$ to $\sim 2500 \mathrm{~nm}$ of the pure [Fig. 7(a)], Wdoped [Figs. 7(b)-7(d)], and the Tb-doped [Figs. 7(e)-7(g)] $\mathrm{VO}_{2}$ thin films are used to calculate the solar transmittance, $T_{\text {sol }}$. For the luminous transmittance, $T_{\text {lum }}$, calculations in
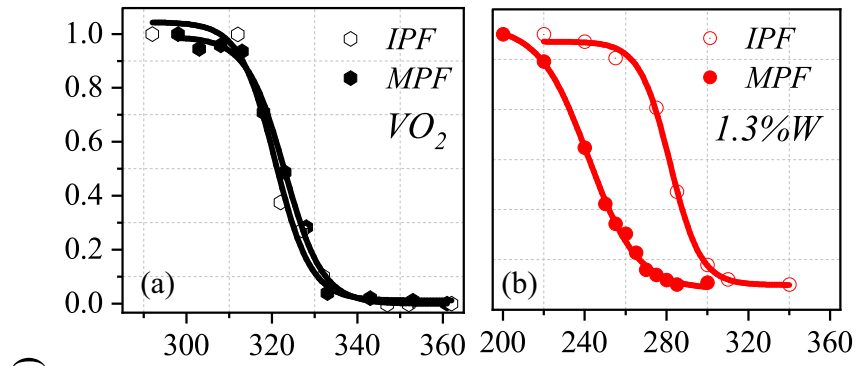

矛
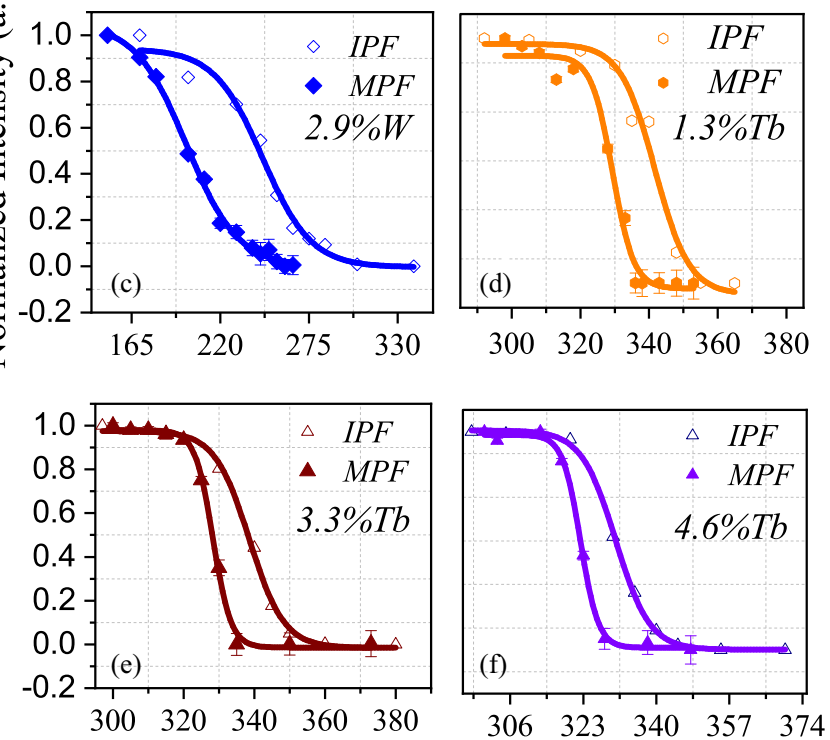

Temperature $(\mathrm{K})$

FIG. 6. (a)-(f) Temperature-dependent variation of the insulating phase fraction (IPF) obtained from optical transmittance measurements and monoclinic phase fraction (MPF) obtained through the $\omega_{0}$ Raman mode in the pure and the $\mathrm{W}$-/Tb-doped $\mathrm{VO}_{2}$ thin films. Data shown in these graphs are from the cooling cycles.

the spectral range $\sim 382 \mathrm{~nm}$ to $\sim 732 \mathrm{~nm}$ are used. Both the solar and the luminous transmittance are calculated using the following formula:

$$
T_{\text {sol/lum }}=\frac{\int \phi_{\text {sol/lum }}(\lambda) T_{r}(\lambda) d \lambda}{\int \phi_{\text {sol/lum }}(\lambda) d \lambda}
$$

where $T_{r}(\lambda)$ is the spectral transmittance at wavelength $\lambda$. $\phi_{\text {sol }}(\lambda)$ is the solar spectral irradiance for the sun standing $37^{\circ}$ above the horizon (for the air mass 1.5 ) and $\phi_{\text {lum }}$ is the standard luminous efficiency function for the photopic vision of human eyes [6,32]. Using the integrated solar and luminous transmittance, we can calculate the solar and luminous modulation of the pure and doped $\mathrm{VO}_{2}$ thin films using the following formulas:

$$
\begin{gathered}
\Delta T_{\mathrm{sol}}=T_{r(\mathrm{sol}, I)}-T_{r(\mathrm{sol}, M)}, \\
\Delta T_{\operatorname{lum}}=T_{r(\operatorname{lum}, I)}-T_{r(\operatorname{lum}, M),}
\end{gathered}
$$

where $T_{r(\mathrm{sol}, I)}, T_{r(\mathrm{sol}, M)}, T_{r(\mathrm{lum}, I)}$, and $T_{r(\mathrm{lum}, M)}$ are the solar and the luminous transmittance of the insulating and the metallic states, respectively. Parameters obtained from transmittance spectra of the pure and the $\mathrm{W}$-/Tb-doped $\mathrm{VO}_{2}$ thin films 


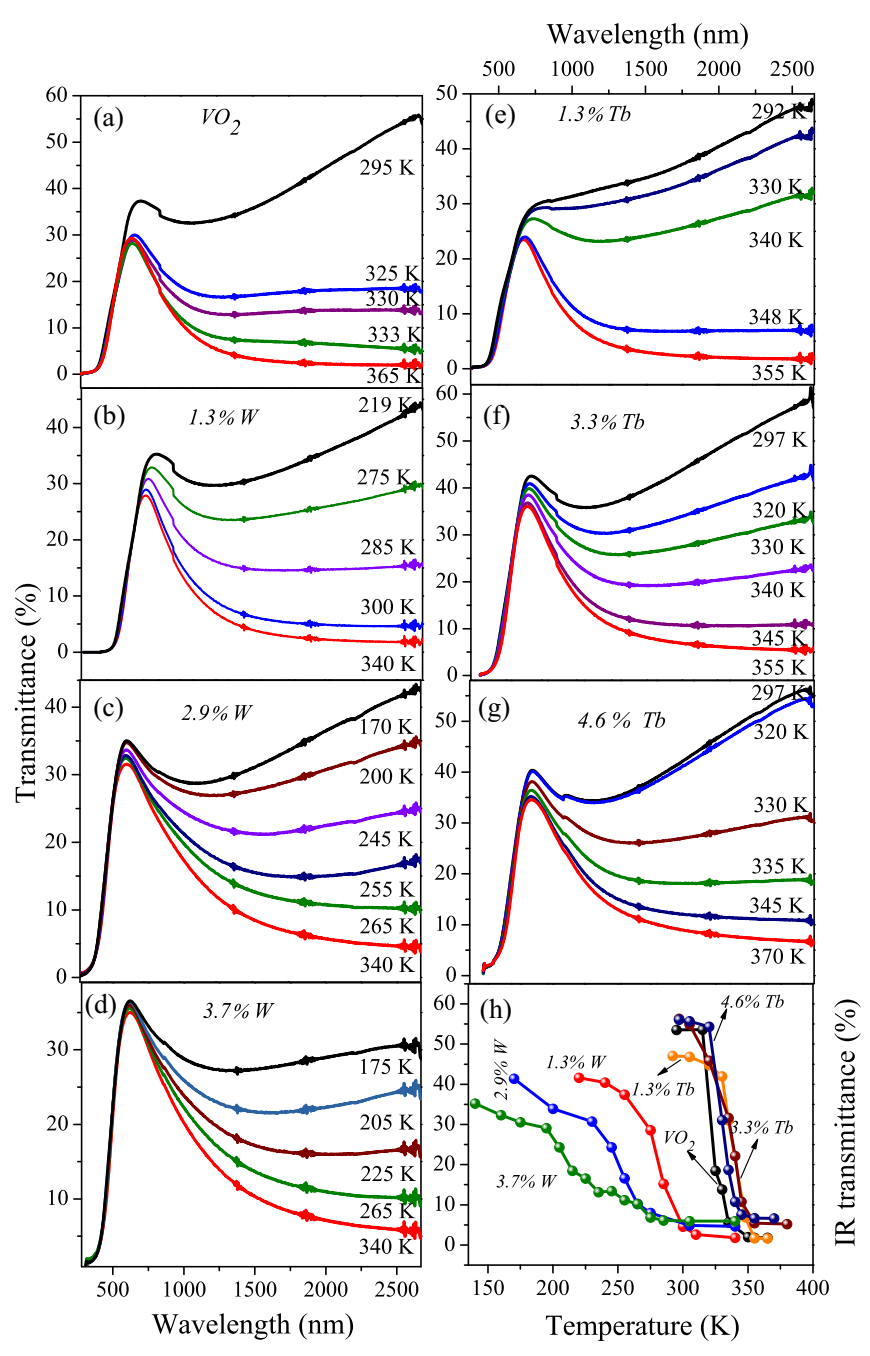

FIG. 7. (a)-(g) Temperature-dependent optical transmittance spectra of the pure and $\mathrm{W}$-/Tb-doped $\mathrm{VO}_{2}$ thin films. (h) Temperature-dependent IR transmittance at $\sim 2500 \mathrm{~nm}$ of the pure and $\mathrm{W}$-/Tb-doped $\mathrm{VO}_{2}$ thin films in the cooling cycles.

are illustrated in Table II. IR switching in the pure and the $\mathrm{W}$-/Tb-doped $\mathrm{VO}_{2}$ thin films can be visualized from Fig. 7(h), where temperature-dependent change in the IR transmittance at the wavelength, $\lambda \sim 2500 \mathrm{~nm}$ is plotted in the cooling cycle. The IR switching transition temperatures for the pure and the $\mathrm{W}$-/Tb-doped $\mathrm{VO}_{2}$ thin films are calculated from the

TABLE II. Parameters obtained from the temperature-dependent optical transmittance measurements of the pure and $\mathrm{W}$-/Tb-doped $\mathrm{VO}_{2}$ thin films.

\begin{tabular}{lcccccr}
\hline \hline Sample & $T_{\text {lum }, \mathrm{I}}$ & $T_{\text {lum }, \mathrm{M}}$ & $\Delta T_{\text {lum }}$ & $T_{\text {sol, } \mathrm{I}}$ & $T_{\text {sol, } \mathrm{M}}$ & $\Delta T_{\text {sol }}$ \\
\hline $\mathrm{VO}_{2}$ & 20.8 & 19.5 & 1.3 & 32.4 & 17.0 & 15.4 \\
$1.3 \% \mathrm{~W}$ & 20.6 & 19.4 & 1.2 & 30.2 & 16.4 & 13.7 \\
$2.9 \% \mathrm{~W}$ & 28.1 & 26.4 & 1.67 & 32.7 & 23.4 & 9.2 \\
$3.7 \% \mathrm{~W}$ & 30.9 & 30.8 & 0.07 & 32.6 & 26.6 & 5.9 \\
$1.3 \% \mathrm{~Tb}$ & 17.1 & 14.9 & 2.2 & 28.9 & 13.6 & 15.2 \\
$3.3 \% \mathrm{~Tb}$ & 28.8 & 26.1 & 2.6 & 38.9 & 23.7 & 15.1 \\
$4.6 \% \mathrm{~Tb}$ & 31.4 & 27.9 & 3.5 & 38.8 & 25.4 & 13.4 \\
\hline \hline
\end{tabular}

IR transmittance vs $T$ curve, measured in the cooling cycle, using Eq. (1), and is found equivalent to $T_{o c}$ (see Table I). The near RT $T_{o c}, T_{r(\text { lum })} \geqslant 40 \%$, and the $\Delta T_{\text {sol }} \geqslant 10 \%$ are considered the best criteria for the $\mathrm{VO}_{2}$ thin films to be employed in thermochromic windows applications [6,33]. From Table II, it is found that the $\mathrm{W}$ doping in the $\mathrm{VO}_{2}$ thin film effectively reduces the IR switching temperature to near RT $\sim 282 \mathrm{~K}$ (measured in the cooling cycle) but is accompanied with a decrease in the solar modulation ability, consistent with earlier reports [16,34]. With the Tb doping, IR switching occurs at higher $T_{o c}$, while the solar modulation ability is maintained around $\sim 15 \%$ which is much large compared to the earlier report on Tb-doped $\mathrm{VO}_{2}$ thin films [15]. There is always a trade-off between the solar modulation ability and the luminous transmittance in the $\mathrm{VO}_{2}$ thin films, i.e., an increase in the luminous transmittance is accompanied by the decrease in the solar modulation [16,17]. However, in the $\mathrm{Tb}$-doped $\mathrm{VO}_{2}$ thin films, increase in the luminous transmittance is accompanied with the good and stable solar modulation ability (see Table II).

To understand the transmittance observations, it is essential to calculate the band gap of the pure and $\mathrm{W}$-/Tb-doped $\mathrm{VO}_{2}$ thin films corresponding to the visible and the IR transparency, which can be extracted from the Tauc plot shown in Figs. 8(a) and 8(b). The Tauc plot is a graphical description between $(\alpha h v)^{n}$ vs $h v$ obtained from the Tauc's equation [35] given as

$$
(\alpha h v)^{n}=A\left(h v-E_{g}\right),
$$

where $n=1 / 2,1 / 3,2$, and 2/3 for the indirect allowed, indirect forbidden, direct allowed and direct forbidden optical transitions, respectively, $\alpha$ is the absorption coefficient obtained from the transmittance spectra, A is a constant, and $h v$ is the photon energy. Usually, in the optical experiments, direct band gap is visualized because of the lower excitation probability among different symmetry points during the optical transition [36]. The band gap is obtained by extrapolating the linear portion of the curve while the $y$ ordinate is zero. Two types of band gaps are observed, i.e., $E_{g 1}$ and $E_{g 2} . E_{g 2}$ corresponds to energy gap between the $\mathrm{V} 3 d_{\|}$and $\pi^{*}$ bands while the $E_{g 1}$ is due to the optical transition between the O $2 p$ and $\pi^{*}$ bands [37,38] [Figs. 8(a) and 8(b)]. The IR switching temperature in all the thin films is found to depend on the energy band gap $E_{g 2}$. The lowering of the IR switching temperature in the $\mathrm{W}$-doped $\mathrm{VO}_{2}$ thin films (see Table II) is attributed to a decrease in the energy band gap, $E_{g 2}$, from $\sim 0.63 \mathrm{eV}$ in the pure to $\sim 0.24 \mathrm{eV}$ in the $1.3 \% \mathrm{~W}$-doped $\mathrm{VO}_{2}$ thin film, while in the Tb-doped $\mathrm{VO}_{2}$ thin films we have observed the highest IR switching temperature $\sim 341.6 \mathrm{~K}$ in the $\sim 1.3 \%$ Tb-doped $\mathrm{VO}_{2}$ thin film having the largest band gap $E_{g 2} \sim 0.74 \mathrm{eV}$.

The band gap $E_{g 1}$ corresponds to the luminous transparency of the thin films, the higher the $E_{g 1}$ value, the higher the visible transparency will be [37-39]. Figures 8(a)(ii) and 8(b)(ii) show the variation of the luminous transmittance along with the absorption edge $\left(\lambda_{K}\right)$ obtained from the derivative of the transmittance spectra. The blueshift in the absorption edge along with an increase in the luminous transmittance in the $\mathrm{VO}_{2}$ thin film due to $\mathrm{W}$ and $\mathrm{Tb}$ doping are found to be consistent with an increase in the energy band-gap values $E_{g 1}$. 

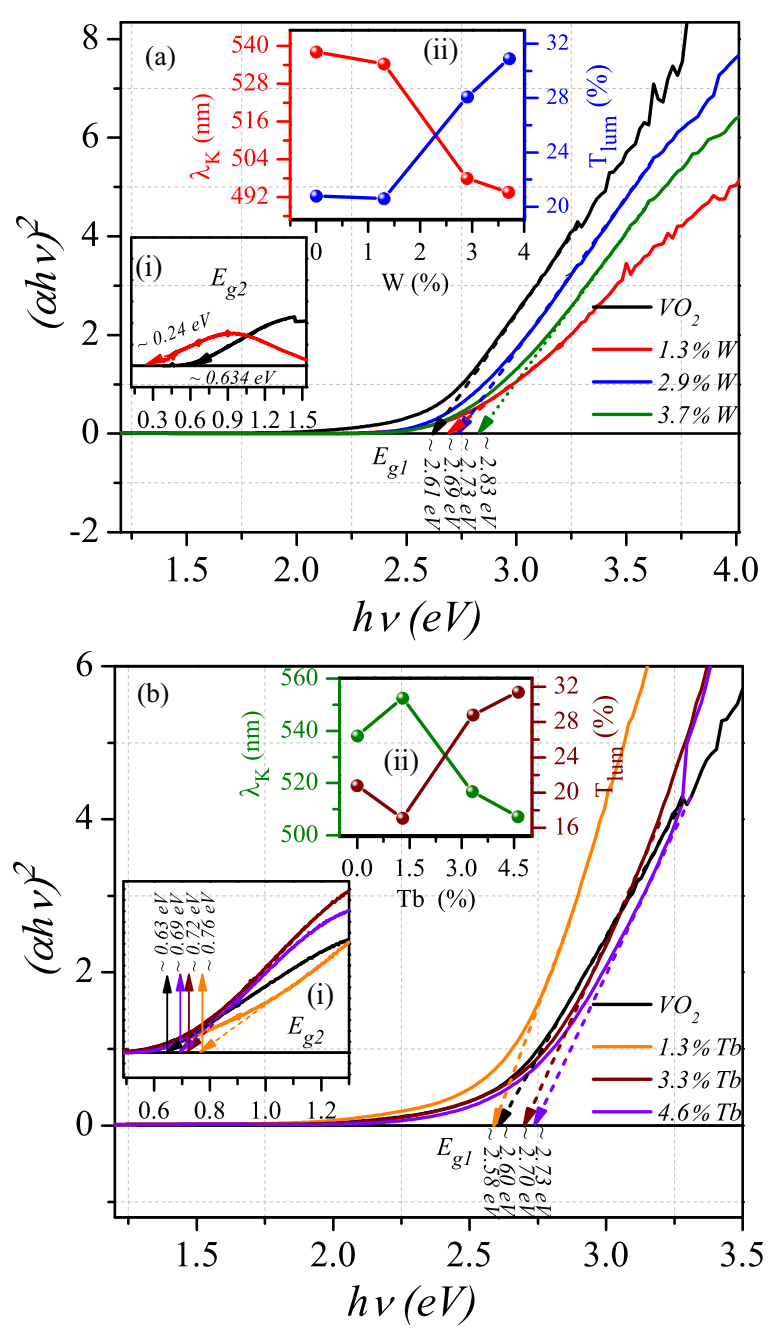

FIG. 8. (a) Tauc plot $\left[(\alpha h v)^{2}\right.$ vs $\left.h v\right]$ of the pure and W-doped $\mathrm{VO}_{2}$ thin films at room temperature. Insets: (i) Tauc plot in the IR range of photon energies and (ii) variation in the $T_{\text {lum }}$ and the $\lambda_{K}$ with the W-doping percentage. (b) Tauc plot of the pure and Tb-doped $\mathrm{VO}_{2}$ thin films at room temperature. Insets: (i) Tauc plot in the IR range of photon energies and (ii) variation in the $T_{\text {lum }}$ and the $\lambda_{K}$ with the Tb-doping percentage.

In the following, we will discuss the origin of dopinginduced changes in the electrical conductivity and optical properties of $\mathrm{VO}_{2}$. The $\mathrm{V} L_{2,3}$ edge x-ray absorption spectra of the pure and the $\mathrm{W}-/ \mathrm{Tb}$-doped $\mathrm{VO}_{2}$ thin films are shown in Fig. 9(a). The vanadium $L_{2,3}$ spectrum arises due to the transition from the V $2 p$ core level (spin orbit split V $2 p_{3 / 2}$ and $2 p_{1 / 2}$ ) to the unoccupied $\mathrm{V} 3 d$ states and consists of two pronounced features at $\sim 518.8 \mathrm{eV}\left(\mathrm{L}_{3}\right)$ and $\sim 525.5 \mathrm{eV}$ $\left(\mathrm{L}_{2}\right)$. The features at $\sim 516.0 \mathrm{eV}$ and $\sim 522.6 \mathrm{eV}$ are due to the transitions to the crystal field split $\mathrm{t}_{2 g}$ part of the $\mathrm{V} 3 d$ states [40,41]. The energy positions of the $L_{3} \sim 518.8 \mathrm{eV}$ and $L_{2} \sim 525.5 \mathrm{eV}$ features confirm $4+$ oxidation state of the vanadium. With the $\mathrm{W}$ and $\mathrm{Tb}$ doping in the $\mathrm{VO}_{2}$ thin film, the slight shift in the energy positions of the $L_{3}$ and $L_{2}$ states is observed, which is due to the additional $\mathrm{V}^{3+}$ and $\mathrm{V}^{4+}$ states created by the $\mathrm{W}$ and $\mathrm{Tb}$ doping, as inferred from the XPS measurements also.
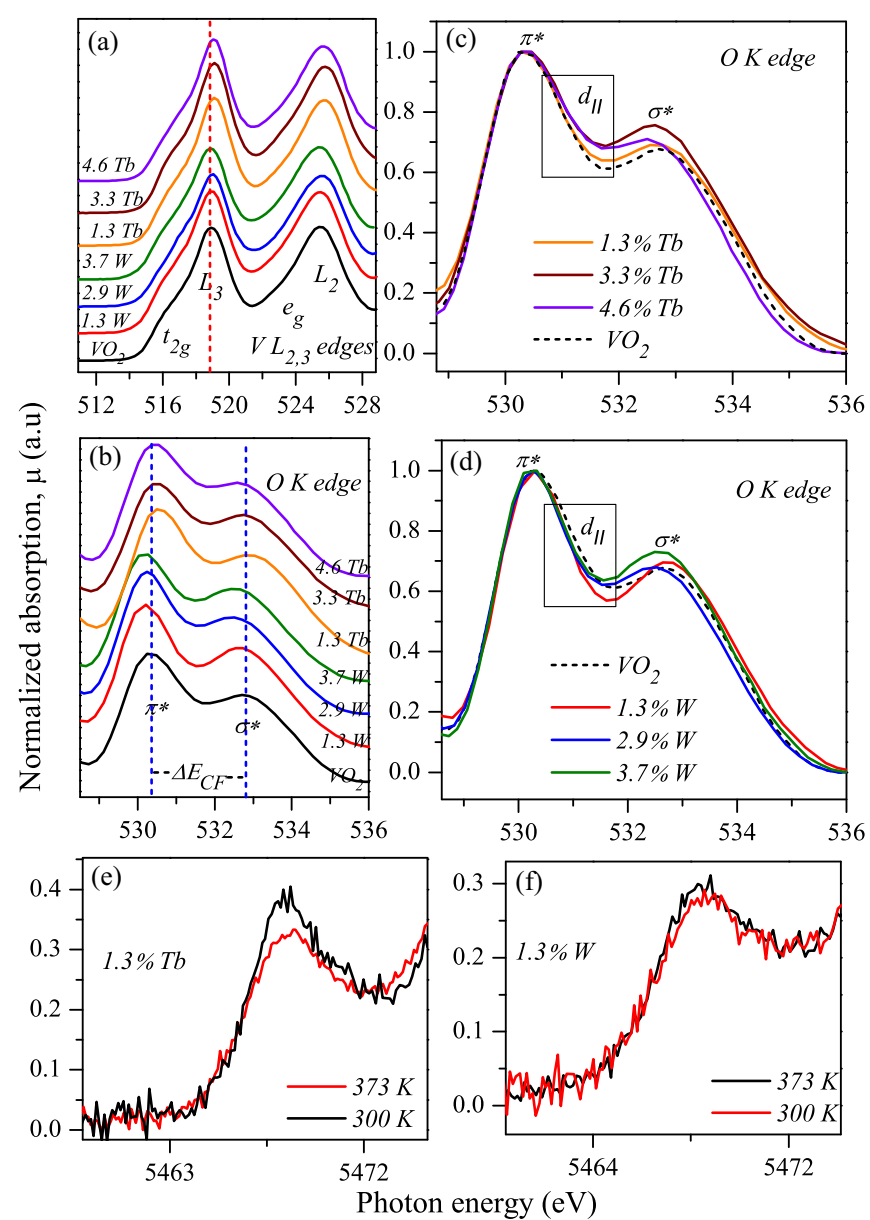

FIG. 9. Room temperature (a) $\mathrm{V} \mathrm{L}_{2,3}$ (b) $\mathrm{O} K$-edge XAS measurements of pure and $\mathrm{W}$-/Tb-doped $\mathrm{VO}_{2}$ thin films. Normalized $\mathrm{O}$ $K$-edge XAS of (c) pure and Tb-doped, (d) pure and W-doped $\mathrm{VO}_{2}$ thin films. The V $K$-pre-edge XAS (e) of $1.3 \%$ Tb-doped and (f) of $1.3 \% \mathrm{~W}$-doped $\mathrm{VO}_{2}$ thin films at $300 \mathrm{~K}$ and $373 \mathrm{~K}$.

The $O K$-edge spectrum, see Fig. 9(b), results from transition of the $\mathrm{O} 1 s$ electrons to the $\mathrm{O} 2 p$ states which are hybridized with the $\mathrm{V} 3 d$ orbitals and form the conduction band. Peaks at $\sim 530.3 \mathrm{eV}$ and $\sim 532.8 \mathrm{eV}$ are due to transitions into the $\pi^{*}$ and the $\sigma^{*}$ hybridized bands $[8,42]$. Doping of the $\mathrm{W}$ has clearly resulted in a shift of the $\pi^{*}$ band toward the lower energies as compared to the pure $\mathrm{VO}_{2}$ thin film, which signifies the stabilization of the metallic state. The $\mathrm{Tb}$ doping shifts the $\pi^{*}$ band toward higher energy, signifying the stabilization of the insulating state. The crystal-field splitting energy $\left(\Delta \mathrm{E}_{\mathrm{CF}}\right)$ which is the energy separation between the $\pi^{*}$ and the $\sigma^{*}$ states is found to vary with the $\mathrm{W}$ and the $\mathrm{Tb}$ doping. Variations in the crystal-field splitting energy values due to the $\mathrm{W}$ and the $\mathrm{Tb}$ doping in the $\mathrm{VO}_{2}$ thin film are also susceptible to a different extent of $\mathrm{V}-\mathrm{V}$ interactions. The $\pi^{*}$ orbitals in the $\mathrm{VO}_{2}$ thin film point in between the ligands and have less orbital overlap with the $\mathrm{O} 2 p$ orbitals, while the $\sigma^{*}$ orbitals are directed toward the ligand having a higher overlap with the $\mathrm{O} 2 p$ orbitals. Therefore, spectral weight of the $\pi^{*}$ band is highly sensitive to the $\mathrm{V}-\mathrm{V}$ interactions while spectral weight of the $\sigma^{*}$ band is related to the direct V-O interactions [43]. To clearly visualize the spectral changes 
among the XAS spectra of pure and doped $\mathrm{VO}_{2}$ thin films, we have carried out the normalization and horizontal shift of the $\pi^{*}$ states to $\sim 532.8 \mathrm{eV}$ as shown in Figs. 9(c) and 9(d). The asymmetry observed in the $\pi^{*}$ bands around $\sim 531 \mathrm{eV}$, which is marked in both Figs. 9(c) and 9(d), is assigned to the unoccupied $d_{\|}$states, whose spectral weight is related to $\mathrm{V}-\mathrm{V}$ dimerization [43-45]. The decrease and an increase in the intensity of the $\mathrm{d}_{\|}$feature is observed in the $\mathrm{W}$ and Tb doping, respectively, and for better visualization and comparison pure $\mathrm{VO}_{2}$ spectra is shown by the dotted line. In the insulating $\mathrm{VO}_{2}$ phase, we did not observe splitting of the $\mathrm{d}_{\|}$feature, as observed by Koethe et al. [44] during their polarized XAS measurements on $\mathrm{VO}_{2}$ single crystal because the spectral weight of the $d_{\|}$feature measured in the highly oriented $\mathrm{VO}_{2}$ thin films is an averaged $\mathrm{d}_{\|}$intensity of the randomly oriented $\mathrm{VO}_{2}$ crystallites in the plane perpendicular to the surface normal [ [ $\left.\begin{array}{lll}0 & 1 & 1\end{array}\right]$ as the polarization vector of the incident photons was perpendicular to the surface normal during the experiments and probes all the randomly oriented $\mathrm{V} 3 \mathrm{~d}_{\|}$states along the monoclinic $a$ axis (or rutile $c$ axis, as $a_{M}=2 c_{R}$ ). Due to this averaging effect, the splitting of the $d_{\|}$feature is obscured in the $\mathrm{VO}_{2}$ thin films. The integrated intensity of the $\mathrm{d}_{\|}$states for the pure and the doped $\mathrm{VO}_{2}$ thin films is noted in Table I. The pre-edge structures at $\sim 5468.3 \mathrm{eV}$ from the $\mathrm{V} K$-edge XAS observed in $1.3 \% \mathrm{~Tb}$ and $\mathrm{W}$-doped $\mathrm{VO}_{2}$ thin films at the temperatures $330 \mathrm{~K}$ and $373 \mathrm{~K}$ are shown in Figs. 9(e) and 9(f). The pre-edge peak intensity is highly sensitive to the local geometrical structure around the absorbing atom $(\mathrm{V})$ and increases directly with increase in distortion of the regular octahedral $\mathrm{VO}_{6}$ units [18]. The $\mathrm{VO}_{2}$ rutile, $R$ phase has less distorted octahedron compared to the monoclinic, $M 1$ phase, hence an increase in intensity in the $300 \mathrm{~K}$ spectra compared to the $370 \mathrm{~K}$ spectra of the Tb-doped $\mathrm{VO}_{2}$ thin film represent the transition from the $R$ phase to the M1 phase while no such signature is observed in the W-doped $\mathrm{VO}_{2}$ thin film, indicating a stable rutile phase at RT and $373 \mathrm{~K}$. By combining the resistivity and XAS measurements, we find that the IMT strength of the pure and $\mathrm{W}$-/Tb-doped $\mathrm{VO}_{2}$ thin films is directly related to the unoccupied spectral weight of the $\mathrm{V} 3 d_{\|}$states. $\mathrm{W}$ doping is found to decrease the IMT strength while $\mathrm{Tb}$ doping increases the IMT strength which is accompanied by a decrease and an increase in the spectral weight of the $\mathrm{V} 3 d_{\|}$states [see Table I and Figs. 9(c) and 9(d)]. The changes in the IMT strength and the spectral weight of the $d_{\|}$states within the error limits are not systematic with the $\mathrm{W}$ and the Tb dopings, however, we can clearly visualize respective increase and decrease in both the IMT strength and the $d_{\|}$spectral weight with the Tb and $\mathrm{W}$ dopings, as compared to the pure $\mathrm{VO}_{2}$ thin film.

Spectral weight of the $d_{\|}$states observed in the XAS measurements is directly related to the vanadium-vanadium dimerization along the rutile $c$ axis $[44,45]$. The $\mathrm{V}-\mathrm{V}$ dimerization in the $\mathrm{VO}_{2}$ can be directly visualized from the $\omega_{v_{1}}$ and the $\omega_{v_{2}}$ vibrational modes in Raman spectra shown in Figs. 10(a) $-10(\mathrm{~g})$ as both these modes are assigned to V-V vibrations and the $\omega_{v_{1}}$ frequency mode gives the twisting vibration of the vanadium dimers along the rutile $c$ axis. Raman mode $\omega_{v_{1}}$ in the pure and Tb-doped $\mathrm{VO}_{2}$ thin films is fitted with a single Lorentzian while in the $\mathrm{W}$-doped $\mathrm{VO}_{2}$ thin films, the vibrational mode is fitted with two Lorentzian

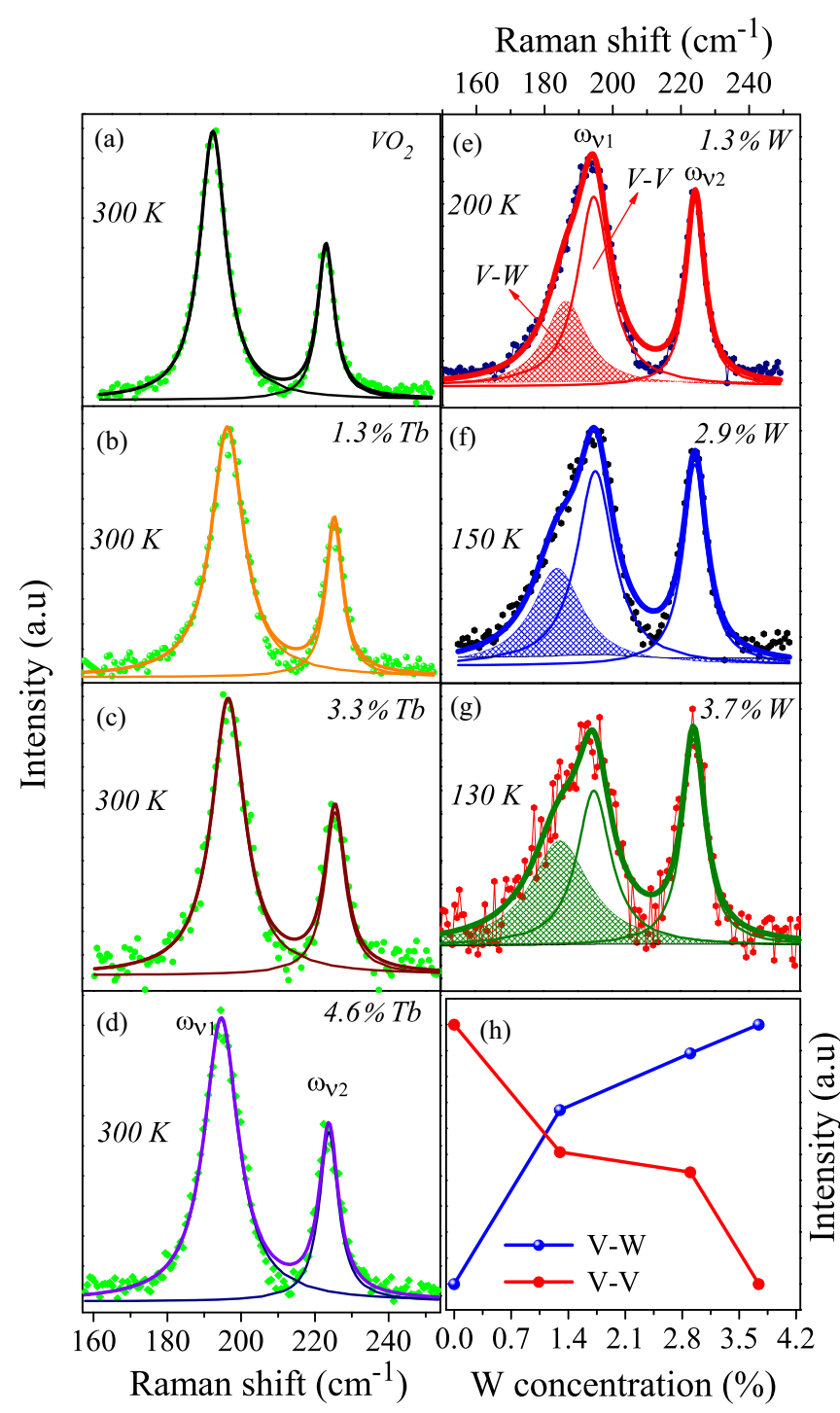

FIG. 10. (a)-(d) Room temperature (300 K) Raman-integrated intensity of the $\omega_{v_{1}}$ and $\omega_{v_{2}}$ vibrational modes in pure and Tb-doped $\mathrm{VO}_{2}$ thin films. (e)-(g) Raman integrated intensity of the $\omega_{v_{1}}$ and $\omega_{v_{2}}$ vibrational modes in the $1.3 \%, 2.9 \%$, and $3.7 \% \mathrm{~W}$-doped $\mathrm{VO}_{2}$ thin films at $200 \mathrm{~K}, 150 \mathrm{~K}$, and $130 \mathrm{~K}$, respectively. (h) Raman intensity variation of the $\mathrm{V}-\mathrm{O}$ and $\mathrm{V}-\mathrm{W}$ modes with the increase in the $\mathrm{W} \%$ concentration.

peaks at $\sim 186$ and $195 \mathrm{~cm}^{-1}$. The additional peak at the lower frequency value $\sim 186 \mathrm{~cm}^{-1}$ is assigned to the $\mathrm{V}-\mathrm{W}$ vibrational mode as effective mass of the $\mathrm{V}-\mathrm{W}$ system is larger as compared to the V-V system. Presence of the V-W vibrational modes in the W-doped $\mathrm{VO}_{2}$ thin films signify replacement of the $\mathrm{V}$ atoms by the doped $\mathrm{W}$ atoms along the $\mathrm{VO}_{2}$ rutile $c$ axis, hence the loss of $\mathrm{V}-\mathrm{V}$ dimerization. On the contrary, in the Tb-doped $\mathrm{VO}_{2}$ thin film absence of any such extra mode signify that the $\mathrm{Tb}$ is not doped along the rutile $c$ axis therefore there is no loss of $\mathrm{V}-\mathrm{V}$ dimerization. Figure $10(\mathrm{~h})$ shows the intensity variation of the V-V and the $\mathrm{V}$-W vibrational modes in the $\mathrm{W}$-doped sample. Increase in intensity of the $\mathrm{V}-\mathrm{W}$ vibrational mode and corresponding decrease in intensity of the $\mathrm{V}-\mathrm{V}$ vibrational mode with increase in the $\mathrm{W}$ doping can be visualized. According to 


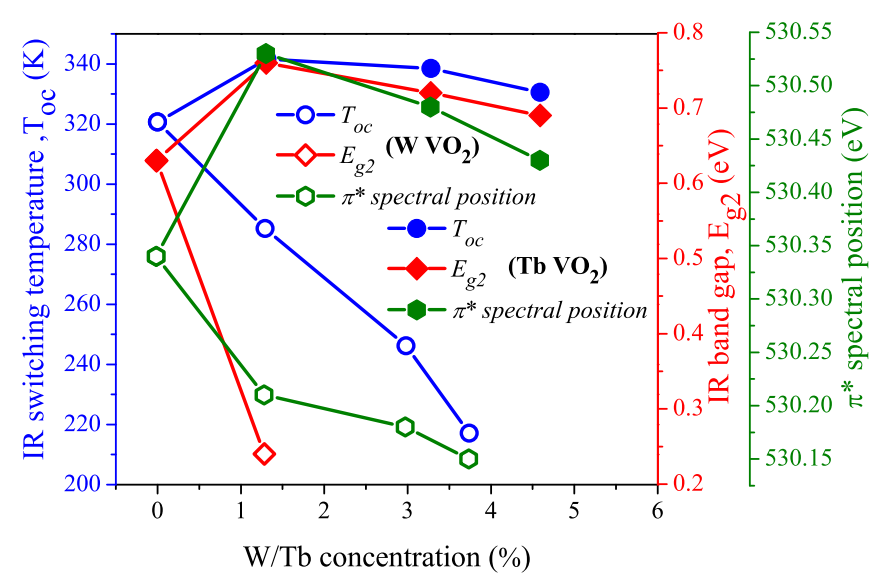

FIG. 11. Variation in the spectral position of the $\pi^{*}$ states, IR band gap $\left(\mathrm{E}_{g 2}\right)$, and the IR switching temperature $\left(T_{o c}\right)$ with the $\mathrm{W} \%$ and the $\mathrm{Tb} \%$ concentration in the $\mathrm{VO}_{2}$ thin films.

Tang et al. [46], electron dopant $\mathrm{W}$ atoms break the $\mathrm{V}^{4+}-\mathrm{V}^{4+}$ dimers and result in formation of the $\mathrm{V}^{3+}-\mathrm{W}^{6+}$ and $\mathrm{V}^{3+}-\mathrm{V}^{4+}$ pairs along the rutile $c$ axis due to electron transfer from the $\mathrm{W}$ to the $\mathrm{V}$ ions. The electronic charge transfer from the $\mathrm{W}$ atoms resulting in formation of $\mathrm{V}^{3+}$ ions (see Fig. 3) stabilizes the metallic state and the loss of the vanadium dimerizations favors the rutile symmetry $[13,46]$.

XAS and optical transmittance results reveal that the IR switching temperature, $T_{o c}$, is strongly related to the spectral weight and position of the $\mathrm{V} 3 d_{\|}$and $\pi^{*}$ states (see Figs. 9 and 11). IR switching temperature $\left(T_{o c}\right)$ in all thin films, which is sensitive to the energy gap $\mathrm{E}_{g 2}$ (energy gap between the $d_{\|}$and the $\pi^{*}$ states), is found to be directly related to the spectral position of the $\pi^{*}$ states (see Fig. 11). The spectral weight and position of the $\pi^{*}$ band is directly sensitive to the V-V interactions and reflects the extent of the V-V hybridization [47]. Larger V-V hybridization will stabilize the $\pi$ bonding states and simultaneously shift the $\pi^{*}$ antibonding states toward the higher photon energy. The decreasing $T_{o c}$ and $E_{g 2}$ with increasing W-doping concentration and increasing $T_{o c}$ and $E_{g 2}$ with increasing $\mathrm{Tb}$ concentration are found to be concomitant with shift of the $\pi^{*}$ states spectral weight toward lower and higher energies, respectively. The solar modulation ability of all the thin films can be directly associated with their IMT strength. In the W-doped $\mathrm{VO}_{2}$ thin films, $\Delta T_{\text {sol }}$ decreases with decrease in the IMT strength. In the Tb-doped $\mathrm{VO}_{2}$ thin films, an increase and the stabilized $\Delta T_{\text {sol }}$ values (contrary to a previous report [15]) can be attributed to an increased IMT strength observed from the resistivity measurements. Hence the solar modulation ability, $\Delta T_{\mathrm{sol}}$, is found to be largely dependent on stabilization of the $\mathrm{VO}_{2}$ monoclinic, $M 1$ phase, and therefore on preservation of the $\mathrm{V}-\mathrm{V}$ dimers.

\section{CONCLUSIONS}

In summary, changes in electrical, structural, optical, and electronic structure properties of the pure and $\mathrm{W}$-/Tb-doped $\mathrm{VO}_{2}$ thin films have been studied. Electron dopant $\mathrm{W}$ atoms are found to significantly reduce the IMT temperature in the $\mathrm{VO}_{2}$ thin film by stabilizing the rutile metallic phase, contrary to the hole dopant $\mathrm{Tb}$ atoms which favor and stabilize the insulating monoclinic phase. Loss of the V-V dimerization is clearly observed due to the $\mathrm{W}$ doping while the $\mathrm{Tb}$ doping is found to strengthen the $\mathrm{V}-\mathrm{V}$ dimerization. Loss of the $\mathrm{V}-\mathrm{V}$ dimerization has been found to directly influence the spectral weight of the $\mathrm{V} 3 d_{\|}$states, a decrease and an increase in spectral weight of the $d_{\|}$states has been observed due to the $\mathrm{W}$ and the Tb doping, respectively. Changes in strength of the IMT, the electrical conductivity, the IR switching temperature, and the solar modulation ability due to the $\mathrm{W}$ and $\mathrm{Tb}$ doping are found directly related to the spectral changes in the $d_{\|}$states. Conclusions which are directly related to thermochromic applications of doped $\mathrm{VO}_{2}$ thin films are as follows: (i) A decrease in the IR switching temperature is observed due to the $\mathrm{W}$ doping while an increase in the IR switching temperature is visualized due to the Tb doping. Near RT, IR switching temperature ( $\sim 285 \mathrm{~K}$, cooling cycle) is observed at an optimum $1.3 \% \mathrm{~W}$ concentration. (ii) An increase in the luminous transmittance is observed for both the $\mathrm{W}$ and the $\mathrm{Tb}$ doping. Luminous transmittance of $\sim 30.9 \%$ and $31.4 \%$ is observed for the highest $3.7 \% \mathrm{~W}$ and $4.6 \% \mathrm{~Tb}$ dopings. (iii) An increased and stabilized solar modulation ability $(\sim 15.2)$ is observed with the $1.3 \% \mathrm{~Tb}$ doping and a decrease in the solar modulation ability $(\sim 13.7)$ due to an optimum $1.3 \% \mathrm{~W}$ doping. Our results also clearly indicate that the $\mathrm{W}$ and the Tb codoping may be a potential candidate to get a combination of lowered IMT temperature, large $\Delta T_{\text {sol }}$, and high $T_{\text {lum }}$ essential for smart window applications.

\section{ACKNOWLEDGMENTS}

Authors are thankful to Sharad Karwal, Rakesh Sah, and Ajay Rathore for help during XPS, XAS, and Raman measurements, respectively. R.J. Choudhary is gratefully acknowledged for providing the PLD setup. E. Welter is gratefully acknowledged for his support during hard x-ray absorption spectroscopy measurements, performed under IndiaDESY collaboration. K.G. thanks Council of Scientific and Industrial Research, New Delhi, India, for support in form of SRF. D.K.S. acknowledges support from SERB, India, in the form of an early-career research award (Award No. ECR/2017/000712).
[1] A. Zylbersztejn and N. F. Mott, Phys. Rev. B 11, 4383 (1975).

[2] M. Imada, A. Fujimori, and Y. Tokura, Rev. Mod. Phys. 70, 1039 (1998).

[3] D. Adler, Rev. Mod. Phys. 40, 714 (1968).
[4] J. Zhou, Y. Gao, Z. Zhang, H. Luo, C. Cao, Z. Chen, L. Dai, and X. Liu, Sci. Rep. 3, 3029 (2013).

[5] C. Wu, F. Feng, and Y. Xie, Chem. Soc. Rev. 42, 5157 (2013). 
[6] L. Zhao, L. Miao, C. Liu, C. Li, T. Asaka, Y. Kang, Y. Iwamoto, S. Tanemura, H. Gu, and H. Su, Sci. Rep. 4, 7000 (2014).

[7] J. B. Goodenough, J. Solid State Chem. 3, 490 (1971).

[8] J. Goodenough and H. Y. Hong, Phys. Rev. B 8, 1323 (1973).

[9] M. K. Dietrich, F. Kuhl, A. Polity, and P. J. Klar, Appl. Phys. Lett. 110, 141907 (2017).

[10] A. Gavini and C. C. Kwan, Phys. Rev. B 5, 3138 (1972).

[11] C. Batista, J. Carneiro, R. Ribeiro, and V. Teixeira, J. Nanosci. Nanotechnol. 11, 9042 (2011).

[12] C. J. Patridge, L. Whittaker, B. Ravel, and S. Banerjee, J. Phys. Chem. C 116, 3728 (2012).

[13] X. Tan, T. Yao, R. Long, Z. Sun, Y. Feng, H. Cheng, X. Yuan, W. Zhang, Q. Liu, C. Wu et al., Sci. Rep. 2, 466 (2012).

[14] Y. Muraoka and Z. Hiroi, Appl. Phys. Lett. 80, 583 (2002).

[15] N. Wang, M. Duchamp, R. E. Dunin-Borkowski, S. Liu, X. Zeng, X. Cao, and Y. Long, Langmuir 32, 759 (2016).

[16] L. Hu, H. Tao, G. Chen, R. Pan, M. Wan, D. Xiong, and X. Zhao, J. Sol-Gel Sci. Technol. 77, 85 (2016).

[17] W. Burkhardt, T. Christmann, S. Franke, W. Kriegseis, D. Meister, B. Meyer, W. Niessner, D. Schalch, and A. Scharmann, Thin Solid Films 402, 226 (2002).

[18] S. S. Majid, D. K. Shukla, F. Rahman, S. Khan, K. Gautam, A. Ahad, S. Francoual, R. J. Choudhary, V. G. Sathe, and J. Strempfer, Phys. Rev. B 98, 075152 (2018).

[19] N. Sepúlveda, A. Rúa, R. Cabrera, and F. Fernández, Appl. Phys. Lett. 92, 191913 (2008).

[20] K. Shibuya and A. Sawa, J. Appl. Phys. 122, 015307 (2017).

[21] C. Marini, E. Arcangeletti, D. Di Castro, L. Baldassare, A. Perucchi, S. Lupi, L. Malavasi, L. Boeri, E. Pomjakushina, K. Conder, and P. Postorino, Phys. Rev. B 77, 235111 (2008).

[22] X.-B. Chen, J. Kor. Phys. Soc. 58, 100 (2011).

[23] Y. Ji, Y. Zhang, M. Gao, Z. Yuan, Y. Xia, C. Jin, B. Tao, C. Chen, Q. Jia, and Y. Lin, Sci. Rep. 4, 4854 (2014).

[24] H. Takami, T. Kanki, S. Ueda, K. Kobayashi, and H. Tanaka, Phys. Rev. B 85, 205111 (2012).

[25] K. Shibuya, M. Kawasaki, and Y. Tokura, Appl. Phys. Lett. 96, 022102 (2010).

[26] R. Zimmermann, R. Claessen, F. Reinert, P. Steiner, and S. Hüfner, J. Phys.: Condens. Matter 10, 5697 (1998).

[27] G. Silversmit, D. Depla, H. Poelman, G. B. Marin, and R. De Gryse, J. Electron Spectrosc. Relat. Phenom. 135, 167 (2004).

[28] J. F. Moulder, W. F. Stickle, P. E. Sobol, and K. D. Bomben, Handbook of X-ray Photoelectron Spectroscopy (Perkin-Elmer Corporation, Eden Prairie, MN, 1992).

[29] Y. Zhang, W. Li, M. Fan, F. Zhang, J. Zhang, X. Liu, H. Zhang, C. Huang, and H. Li, J. Alloys Compd. 544, 30 (2012).
[30] J. M. Atkin, S. Berweger, E. K. Chavez, M. B. Raschke, J. Cao, W. Fan, and J. Wu, Phys. Rev. B 85, 020101(R) (2012).

[31] Y. Kalcheim, N. Butakov, N. M. Vargas, M.-H. Lee, J. del Valle, J. Trastoy, P. Salev, J. Schuller, and I. K. Schuller, Phys. Rev. Lett. 122, 057601 (2019).

[32] L. Kang, Y. Gao, Z. Zhang, J. Du, C. Cao, Z. Chen, and H. Luo, J. Phys. Chem. C 114, 1901 (2010).

[33] S.-Y. Li, G. A. Niklasson, and C.-G. Granqvist, Thin Solid Films 520, 3823 (2012).

[34] C. G. Granqvist, Sol. Energy Mater. Sol. Cells 91, 1529 (2007).

[35] J. Tauc and A. Menth, J. Non-Cryst. Solids 8, 569 (1972).

[36] G.-H. Liu, X.-Y. Deng, and R. Wen, J. Mater. Sci. 45, 3270 (2010).

[37] M. Wan, M. Xiong, N. Li, B. Liu, S. Wang, W.-Y. Ching, and X. Zhao, Appl. Surf. Sci. 410, 363 (2017).

[38] S.-Y. Li, N. R. Mlyuka, D. Primetzhofer, A. Hallén, G. Possnert, G. A. Niklasson, and C. G. Granqvist, Appl. Phys. Lett. 103, 161907 (2013).

[39] J. Zhou, Y. Gao, X. Liu, Z. Chen, L. Dai, C. Cao, H. Luo, M. Kanahira, C. Sun, and L. Yan, Phys. Chem. Chem. Phys. 15, 7505 (2013).

[40] M. W. Haverkort, Z. Hu, A. Tanaka, W. Reichelt, S. V. Streltsov, M. A. Korotin, V. I. Anisimov, H. H. Hsieh, H.-J. Lin, C. T. Chen, D. I. Khomskii, and L. H. Tjeng, Phys. Rev. Lett. 95, 196404 (2005).

[41] J. Zaanen, G. A. Sawatzky, J. Fink, W. Speier, and J. C. Fuggle, Phys. Rev. B 32, 4905 (1985).

[42] D. Ruzmetov, S. D. Senanayake, and S. Ramanathan, Phys. Rev. B 75, 195102 (2007).

[43] D. Ruzmetov, S. D. Senanayake, V. Narayanamurti, and S. Ramanathan, Phys. Rev. B 77, 195442 (2008).

[44] T. C. Koethe, Z. Hu, M. W. Haverkort, C. Schüßler-Langeheine, F. Venturini, N. B. Brookes, O. Tjernberg, W. Reichelt, H. H. Hsieh, H.-J. Lin, C. T. Chen, and L. H. Tjeng, Phys. Rev. Lett. 97, 116402 (2006).

[45] N. F. Quackenbush, H. Paik, M. J. Wahila, S. Sallis, M. E. Holtz, X. Huang, A. Ganose, B. J. Morgan, D. O. Scanlon, Y. Gu, F. Xue, L.-Q. Chen, G. E. Sterbinsky, C. Schlueter, T.-L. Lee, J. C. Woicik, J.-H. Guo, J. D. Brock, D. A. Muller, D. A. Arena, D. G. Schlom, and L. F. J. Piper, Phys. Rev. B 94, 085105 (2016).

[46] C. Tang, P. Georgopoulos, M. E. Fine, J. B. Cohen, M. Nygren, G. S. Knapp, and A. Aldred, Phys. Rev. B 31, 1000 (1985).

[47] D. Ruzmetov and S. Ramanathan, Thin Film Metal Oxides: Fundamentals and Applications in Electronics and Energy (Springer, New York, 2010). 